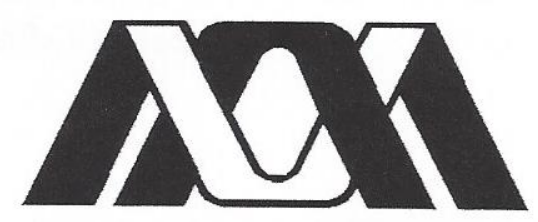

Casa abierta al tiempo

UNIVERSIDAD AUTÓNOMA METROPOLITANA

UNIDAD IZTAPALAPA

DIVISIÓN DE CIENCIAS SOCIALES Y HUMANIDADES

POSGRADO EN CIENCIAS ANTROPOLÓGICAS

La noción de cultura en los trastornos mentales Reflexiones desde la antropología

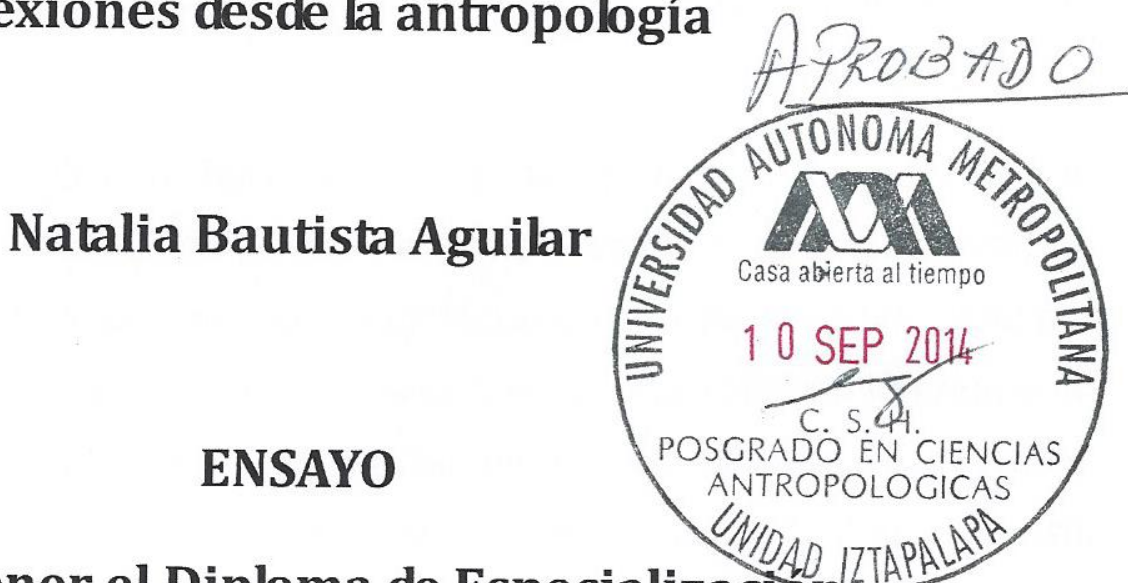

Para obtener el Diploma de Especializacion en Antropología de la Cultura

Director: Dr. Néstor García Canclini

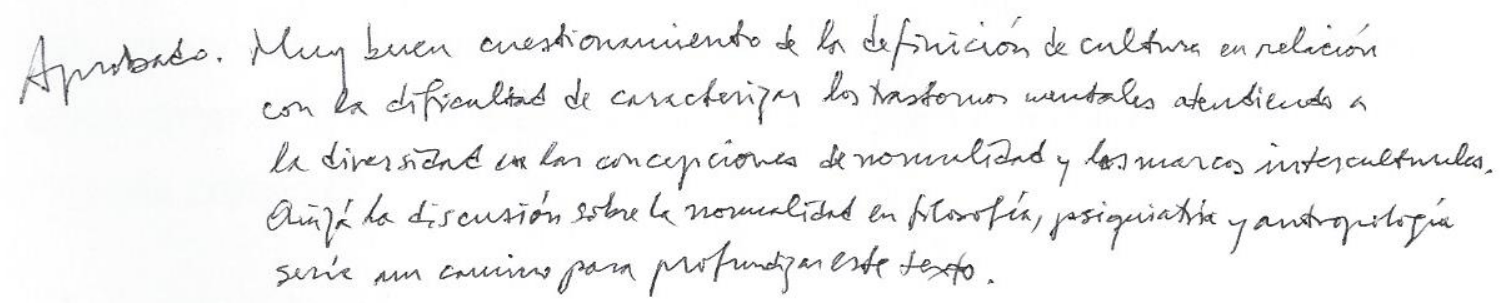

México, D.F.

Junio de 2013 


\section{La noción de cultura en los trastornos mentales Reflexiones desde la antropología}

Cada sistema de pensamiento y nuevo paradigma se configura a la luz de los postulados generados previamente. A partir de su complementación, refutación o reafirmación, cada uno de éstos se va edificando de la mano de transformaciones del orden social, político, económico, histórico y cultural, que tienen lugar en la sociedad que las gesta como una reacción o respuesta a esos mismos, es decir, una serie de adaptaciones o intentos de explicación y apropiación de la realidad vivida. La propia antropología no escapa a tal destino sino que va constituyéndose al interior de la discusión de teorías y postulados, a partir de los cuales rescata, matiza y crea aquellos que le permitirán establecer los paradigmas propios. Al interior de esa discusión el concepto de cultura ha sido central en la definición del objeto/sujeto de estudio de la disciplina antropológica y la particularidad de su enfoque.

Para Baldwin et al. (2006) la historia misma del concepto cultura ha sido controvertida, no sólo por los cambios que ha sufrido resultado de la influencia de fuerzas políticas o económicas, cambios geográficos e ideas prevalentes, sino por el hecho de ser una noción dinámica, vinculada a la continua interacción humana. El estudio de la noción de cultura, implica también enfrentarse a los intereses teóricos y metodológicos de las diversas disciplinas que la han adoptado. De esta forma y atendiendo a lo señalado por Wolf (2003), respecto a que toda formulación de conceptos implica prestar atención al papel del lenguaje dentro de la conformación de ideas y actos. Se vuelve algo fundamental ser conscientes de las diferentes voces que han apelado a la noción de cultura, en la medida que nos permite reconocer la carga histórica-ideológica de su uso, y en determinado momento distanciarse $o$ adherirse a ella, al reformularlos a la luz de los intereses y condiciones presentes. 
Ensayo Especialización

Antropología de la Cultura

Natalia Bautista Aguilar

El objetivo de este ensayo es llevar a cabo una reflexión en torno al papel que se le ha dado a la cultura en la definición, tratamiento y estudio de los trastornos mentales, así como sobre algunos aspectos que persisten y marcan el abordaje predominante en el campo de la investigación transcultural de la salud mental. A fin de identificar algunos desafíos que persisten en el abordaje cultural del proceso salud/enfermedad mental/atención y, con base en ello, señalar la que considero podría ser una perspectiva adecuada para el estudio de la diversidad cultural en los trastornos mentales. Para ello, apoyándome en trabajos que se han dado a la tarea de hacer una revisión de los diversos usos y definiciones del término cultura, destacaré de forma breve y general, los elementos que la caracterizan desde distintas posturas.

De acuerdo con Cuche (2002), si se quiere comprender el sentido que la noción de cultura adquiere en el campo de las ciencias sociales es imprescindible reconstruir su genealogía iniciando por su formación como palabra, seguido de su conformación como concepto científico dando cuenta de su trasformación semántica. De esta forma, retoma el ejemplo de la lengua francesa y señala que la palabra cultura, proveniente del latín "cultura" va de significar un estado (parcela de tierra cultivada), a referir una acción (cultivar). Por su parte, Baldwin et al. (2006) realizan un análisis diacrónico de las distintas acepciones del término cultura, colocando como raíz última de ésta al latín "colere" cuyo significado es "labrar" o "cultivar la tierra". Es decir, el proceso de "cultivar la tierra" habría sido trasladado al proceso de formación de individuos en términos de cultivo de facultades o capacidades humanas; en ese sentido, se explica la evolución semántica del término a partir de un movimiento por metonimia cuando va de la cultura como "estado" a la cultura como "acción", y por metáfora, en tanto que del "cultivo de la tierra" pasa al "cultivo del espíritu" (Cuche, 2002). Por su parte, Williams (1983 cit. en Baldwin et al., 2006) sostiene tres amplias categorías respecto a su uso: la primera de ellas, referente al cultivo como proceso general de desarrollo espiritual, estético e intelectual de grupos o individuos; la segunda, alusiva a la particular forma de vida de la humanidad en general y; una tercera, 
Ensayo Especialización

Antropología de la Cultura

Natalia Bautista Aguilar

correspondiente a obras y actividades intelectuales y artísticas, como la más ampliamente empleada. Igualmente, Kroeber y Kluckhohn (1952 cit. en Baldwin, 2006), ubican el mismo número de tendencias en el uso del concepto, especificando que en principio correspondería a una primer etapa en la que se alude a un tipo de historia general; seguida por la referencia a una cultura ilustrada; y finalmente, la tendencia posterior a 1850 bajo la que se define como el modo característico de la existencia humana. Hay entonces, un desarrollo y uso del concepto cultura en términos de progreso, refinamiento, educación, evolución, que al referirse a lo colectivo establece una asociación con el término "civilización". Se habla de la naturalización de un conjunto de conocimientos y gustos, correspondientes a Occidente, como los únicos válidos de ser difundidos (García Canclini, 2004).

Baldwin et al. (2006) resaltan la raíz germana "kultur" que designa el desarrollo agrícola, como aquella de la que emerge el sentido más ampliamente reconocido y empleado, y de la que se desprende la conceptualización adoptada por la antropología. En cuanto a la revisión del término kultur, Cuche (2002) sitúa a diferencia del caso francés, el establecimiento de una relación de oposición entre cultura y civilización de acuerdo al sistema de valores al que hace referencia. De ahí que la califique como una noción particularista tendiente a la delimitación de diferencias nacionales, de una cultura propia que a su vez designa un patrimonio nacional; mientras que en Francia, la noción de cultura alcanza un desarrollo orientada por la idea de unidad en un sentido más amplio, universalista, con la consecuente minimización de la particularidad. De modo que el debate entre ambas posturas, se concibe arquetípico de las dos formas de concebir la cultura, la particularista y la universalista.

Con relación a la forma universalista, la historia de la evolución de la palabra deja atrás un uso cotidiano para adquirir un carácter científico expresado por la primera definición del concepto de cultura en el campo de la etnología, elaborada por Tylor, como: "el conjunto complejo que incluye el conocimiento, las creencias, el 
Ensayo Especialización

Antropología de la Cultura

Natalia Bautista Aguilar

arte, la moral, el derecho, las costumbres y las otras capacidades o hábitos adquiridos por el hombre en tanto miembro de la sociedad" (Tylor, 1871 cit. en Cuche, 2002: 20). Para algunos antropólogos, este concepto llega a ser considerado el menos colonialista que ha creado Occidente en tanto que a través de él, se reconoce la capacidad de cultura a etnias y grupos no occidentales, o bien, se señala como un concepto que intentaba "reconocer la dignidad de los excluidos" (García Canclini; 1982: 28); al conducir al reconocimiento de la capacidad de cultura como algo no restringido a ciertos grupos o clases sociales.

No obstante, más allá de que la definición de Tylor abre la posibilidad de reconocimiento universal de la capacidad de cultura, es importante señalar que constituye una definición puramente descriptiva y objetiva (Cuche, 2002), que se inscribe en un marco de referencia teórico evolucionista que postula un proceso de evolución lineal por el que se consideraba todas las sociedades debían pasar. Dicho esquema evolutivo estaba conformado por una serie de estadios jerarquizados de acuerdo al grado de avance mostrado (salvajismo-barbariecivilización), lo que a su vez, impulsó la reflexión sobre la presencia de ciertos rasgos culturales similares entre distintas sociedades, un paralelismo que llevaba a considerar el contacto entre culturas y la consecuente difusión de elementos. El difusionismo se presentaba como una alternativa al esquema unilineal, sin embargo, seguía siendo considerado como un "enfoque museográfico de los hechos culturales, reducidos a rasgos que se coleccionan y que se describen por sí mismos sin que sea posible comprender su lugar en el sistema global" (Cuche, 2002:41).

Un aspecto a resaltar, es que para el momento de aparición de la noción de cultura de Tylor, la recolección de materiales y el análisis de éstos, constituían dos aspectos separados, es decir, se encontraban fragmentados dado que la recolección del dato etnográfico no era un trabajo asociado a la práctica del antropólogo. Éste, podía elaborar su análisis y postulados a partir del ordenamiento y estudio de materiales ya recabados por otros que, incluso 
Ensayo Especialización

Antropología de la Cultura

Natalia Bautista Aguilar

provenían de otras disciplinas. De esta forma, el paso de una definición universalista a una particularista, implicó además el cambio de visión del propio trabajo de campo. Para Boas (cit. en Cuche, 2002), la reflexión antropológica debía enfocarse a la diferencia desde una perspectiva distinta a la racial, mostrando a través de sus propios estudios la inexistencia de caracteres raciales inmutables. Si bien, retoma la idea de cultura tyloriana, Boas no apuntaba al descubrimiento de leyes generales o universales de evolución, en tanto que éstas salían del marco de lo que podía ser demostrado empíricamente. De ahí que no sólo sea considerado el fundador de la perspectiva particularista sino del método etnográfico mismo (Cuche, 2002). La perspectiva relativista adoptada por Boas (cit. en Cuche, 2002), consideraba que la interpretación de una cultura no debía ser realizada mediante la aplicación de las categorías del investigador sino que por el contrario, debía partir del examen del sistema cultural en cuestión, de su especificidad.

A diferencia del desarrollo que alcanza la noción de cultura en la antropología norteamericana, en lo que respecta al contexto francés, el desarrollo de la etnografía permaneció por cierto tiempo reducido a la perspectiva sociológica y con ello, un predominio en el uso del concepto de civilización más que de la noción de cultura. En el caso de Durkheim (cit. en Cuche, 2002), reconocido como sociólogo con orientación antropológica, su interés se concentraba en lo social, que incluía como una de sus dimensiones a lo cultural, teniendo que si bien, reconocía la unidad de la humanidad se oponía a las teorías que sostenían una evolución unilineal. En este sentido, su concepto de normalidad social se señala como aquel en que se puede identificar una cierta tendencia a la relatividad cultural en tanto que es postulado en relación a las particularidades de cada sociedad y su nivel de desarrollo (Cuche, 2002). Para Durkheim lo social no sólo precedía al individuo sino se le imponía y trascendía, con lo que dotaba de superioridad a la conciencia colectiva, formada por representaciones colectivas, valores y sentimientos comunes a todos los miembros de una misma sociedad. De acuerdo con Cuche (2002), dando continuidad al intento por pensar la alteridad, 
Ensayo Especialización

Antropología de la Cultura

Natalia Bautista Aguilar

otro de los conceptos que en el marco de la etnología francesa, fue empleado para explicarla en oposición a un modo único de funcionamiento, fue el de "mentalidad" de Lévy-Bruhl. Para éste, no era válido sostener la presencia de las mismas leyes de la razón en todas las sociedades, ya que entre los grupos había variación en el modo de ejercer su pensamiento; por tanto, a través del concepto de mentalidad, más que aludir a los sistemas de representaciones y los modos de razonamiento en el seno de una misma cultura como conjuntos perfectamente estables y homogéneos, esperaba indicar una orientación general de cierta cultura determinada (Cuche, 2002). No obstante, no hay un desarrollo posterior de dicho concepto.

Como puede notarse, la discusión en torno a la noción de cultura continúa permeada por el esquema evolucionista, en tanto perspectiva universalista frente a la cual se proponen distintos abordajes de la diferencia o unidad, tanto social como cultural. Lo anterior lleva a su vez a la aparición de esfuerzos por lograr una diferenciación entre sociedad y cultura, que marcan el desarrollo de ambos conceptos. Kluckhohn (cit. en Baldwin et al., 2006), señala la necesidad de tal distinción reduciendo la "sociedad" al conjunto de personas que comparten e interactúan en un mismo espacio social pero que no comparten una forma de vida, en cuyo caso debiera ser identificado como "cultura". Por su parte, Malinowski, postula a la cultura como un sistema coherente cuyos elementos están en armonía entre sí y poseen un carácter funcional. Tal carácter, es explicado a partir de la satisfacción de necesidades de modo que la cultura quedaría conformada por las respuestas a las necesidades del hombre, sean fisiológicas o derivadas de las mismas, donde dichas respuestas corresponderían a las instituciones creadas por el grupo (Cuche, 2002). De este modo, a diferencia de Kluckhohn, lo social y lo cultural en Malinowski estarían estrechamente relacionados, en tanto que lo cultural adquiere significación en lo social.

Ahora bien, en el marco de la antropología norteamericana, la herencia boasiana culmina en la generación de distintas perspectivas de análisis de la cultura, 
Ensayo Especialización

Antropología de la Cultura

Natalia Bautista Aguilar aquella que prioriza la dimensión histórica, la que conceptualiza a la cultura como un sistema de comunicación y finalmente, la que establece un diálogo con la psicología. La importancia concedida al aspecto psicológico dividió a los discípulos de Boas, para algunos la reflexión cultural debía dirigirse a los comportamientos comunes en tanto que éstos referían a estilos determinados por la cultura. La serie de conductas y actos sostenidos por los miembros de un grupo contendrían de tal modo, la especificidad del mismo. Bajo la forma de la escuela denominada cultura y personalidad, se presentan diversas propuestas que no debieran ser abordadas como un referente teórico unificado bajo la denominación de culturalismo (Cuche, 2002). Así se tiene que, Benedict (cit. en Cuche, 2002) plantea la definición de "tipos culturales" como formas coherentes de organización de rasgos culturales, esquemas inconscientes, ofrecidos a los individuos por una cultura que persigue un objetivo. Mientras que por su parte, Margaret Mead (cit. en Cuche, 2002) se da a la tarea de indagar la relación entre cultura y formación de personalidad cuyos hallazgos, si bien, confirman a la cultura como el sistema de valores, modelos y pautas de comportamiento transmitidos a los miembros del grupo a través de la educación, alude a una noción de cultura que no es ajena a su apropiación, a la reinterpretación individual, es decir, que no es estática. En esta misma línea, Cuche (2002) menciona que en contraposición a lo que se califica de reduccionismo en Benedict, Linton a través de su concepto de "personalidad base" indica que en cada cultura pueden encontrarse distintos tipos de personalidad privilegiados que serán tomados como los "normales", llamando la atención sobre la diversidad al interior del propio grupo en términos de estatus y roles sociales.

Hasta aquí puede distinguirse en el proceso de definición de la cultura, el desplazamiento de un enfoque que inicia con la identificación de artefactos, a uno que poco a poco va integrando otra serie de elementos y conceptos como los referentes al comportamiento y la educación. De acuerdo con Ortner (1993) la antropología cultural y psicocultural norteamericana, junto con el estructuralfuncionalismo británico y la antropología evolucionista norteamericana constituían, para fines de los años cincuenta, los tres grandes paradigmas que caracterizaban 
Ensayo Especialización

Antropología de la Cultura

Natalia Bautista Aguilar

el horizonte teórico de la antropología. A partir de los cuales sería impulsada una combinación de nuevas ideas que en la década siguiente, darían lugar a tres movimientos: la antropología simbólica, la ecología cultural y el estructuralismo.

En la antropología simbólica bajo la figura de Geertz, la cultura queda definida como aquello que "denota un esquema históricamente transmitido de significaciones representadas en símbolos, un sistema de concepciones heredadas y expresadas en formas simbólicas por medios con los cuales los hombres comunican, perpetúan y desarrollan su conocimiento y sus actitudes frente a la vida" (Geertz; 1973: 88). De ahí que el interés se concentrara; más que un estudio del símbolo en sí mismo, en la forma en cómo éstos, modelan las maneras en que los actores sociales ven, sienten y piensan acerca del mundo, es decir, la manera en que operan como vehículos de "cultura" (Ortner, 1993).

Para Geertz (1973 cit. en Ortner, 1993), la cultura no es un sistema ordenador en abstracto, que derive su lógica de recónditos principios estructurales sino de la gente operando dentro de cierto orden institucional e interpretando sus situaciones para actuar coherentemente con ellas, de ahí que el punto de vista del actor sea resaltado como un elemento fundamental para el análisis. Al interior de la antropología simbólica vemos de nuevo como subyace el referente sociedadcultura, en torno al que ha girado gran parte del esfuerzo por definir y estudiar esta última. Si bien, para Geertz, el peso se coloca en los símbolos como vehículos de cultura, para Turner (1967 cit. en Ortner, 1993), debieran ser considerados operadores en el proceso social, elementos que producen transformaciones sociales. Los símbolos cobran interés al interior del ritual en tanto hacen pasar a los actores de una condición a otra y resuelven contradicciones sociales, encadenándolos a categorías y normas de su sociedad. Inaugurando así el sentido pragmático de los símbolos.

Otro gran referente en el que se ha inscrito la definición de cultura es justamente el que marca su oposición con la naturaleza. Esta última durante una parte del desarrollo antropológico fue abordada en términos de condiciones ambientales 
Ensayo Especialización

Antropología de la Cultura

Natalia Bautista Aguilar específicas que daban lugar a formas particulares de organización social (ecología cultural). La cultura subordinada a su relación e intercambio con el medio ambiente, la forma específica de adaptación a dichas condiciones, la explotación y control de los recursos con la consecuente invención tecnológica. Sin embargo, es con Lévi-Strauss (1983) con quien se considera el problema de distinción entre cultura y naturaleza queda resuelto al establecer como criterio de diferenciación la ausencia/presencia de reglas invariables de naturaleza inconsciente que conforman los universales de la cultura, atributos distintivos de la condición humana que se expresan en manifestaciones variadas y diversas de dicha condición compartida, aunque sin agotarla. De acuerdo con Cuche (2002) en el planteamiento de Lévi-Strauss, pueden encontrarse cuatro ideas de Benedict, la primera de ellas correspondería a la noción de modelo que define a cada cultura. La segunda idea sería la que establece como limitados los tipos culturales; la tercera, relativa al estudio de las culturas primitivas como el método más fértil para el establecimiento de las combinaciones de elementos culturales posibles y finalmente, el hecho de que el estudio de dichas combinaciones podía darse de forma independiente al individuo, el cual no era consciente de las mismas. No obstante, se separa del enfoque culturalista de Benedict, al inclinarse al análisis de los universales o invariables de la cultura. El principal impacto que se le reconoce al estructuralismo se ubica en Inglaterra, en su aplicación al análisis de sociedades y cosmologías particulares, así como en torno a los sistemas clasificatorios, la anomalía y la antiestructura. Sin embargo, se señala la existencia de reacciones en contra de ciertos aspectos que fueron concebidos como problemáticos; el primero de estos fue lo que se consideró una negativa en torno al reconocimiento de la relevancia de la intención subjetiva en el proceso sociocultural, mientras el segundo aspecto, quedó constituido por la también negativa de considerar cualquier impacto significante del "acontecimiento" sobre la estructura" (Ortner, 1993).

En la década posterior, se reconoce la tendencia a homologar la noción de cultura a la ideología. En la teoría marxista no hay un tratamiento propio de la misma sino 
Ensayo Especialización

Antropología de la Cultura

Natalia Bautista Aguilar

en función de su contribución a la dinámica de lucha de clases. Se plantea determinada por las condiciones materiales de existencia, introduciendo la relación dominación/subordinación. Del anterior uso dado al término cultura por parte de los marxistas (cultura capitalista), junto con la denominación de Tylor (cultura primitiva), erróneamente llegaron a ser considerados el origen de la dispersión identificada como "zumbido ensordecedor" (García Canclini, 2004) en torno a la cultura.

En la actualidad, el interés de la antropología ya no se concentra tanto en distinguir su objeto/sujeto de estudio, así como en defender o legitimar su autonomía y estatus científico, sino en renovar su perspectiva de análisis en gran parte empujada por las transformaciones a las que su objeto/sujeto de estudio y los contextos en los que se insertan, se enfrentan. Si algo es perfectamente identificado a lo largo del proceso de definición de la noción de cultura ha sido la existencia de un marcado disenso que no logra ser subsanado ni con la construcción de una definición única, pretendidamente completa, emergida de una síntesis de los diversos aspectos o tipos de definición como la que en su momento Kroeber y Kluckhohn (1952 cit. en Baldwin, 2006), a raíz de su revisión, nos brindaran. De acuerdo con García Canclini (2004), la preocupación subyacente es la concerniente a la unicidad y universalidad del conocimiento, en un entorno en que diversidad cultural y diversidad de paradigmas científicos se vuelven coincidentes.

Pero ¿en qué sentido algunos de estos elementos, que caracterizaron en diversos momentos, el uso y definición del concepto cultura, persisten en la configuración de la clasificación de los trastornos mentales?, ¿cuáles son sus efectos, es decir, qué desafíos representan a la investigación en dicho campo?

En el campo de la salud mental, hablar de diversidad cultural, ha llevado a la aplicación de un enfoque frecuentemente dirigido a posibilitar la comparación internacional de la frecuencia de trastornos. De esta forma, gran parte de los esfuerzos y hallazgos se han concentrado en mostrar la presencia y similitud de 
Ensayo Especialización

Antropología de la Cultura

Natalia Bautista Aguilar

los mismos en diferentes sociedades a partir de la aplicación de técnicas estandarizadas de instrumentos diagnósticos (Kleinman, 1987; Martínez-Hernáez, 2006a) Es decir, se prioriza la búsqueda de un patrón uniforme de enfermedad mental reduciendo con ello la heterogeneidad que la cultura aporta a la definición y expresión de la sintomatología. Dicha búsqueda se sostiene bajo una visión de los fenómenos humanos como modelos mecánicos de causa-efecto. Al reconocer el papel determinante de la biología como causa y estructura de los trastornos, la cultura se ve tan sólo como un aspecto secundario que matiza el contenido de los síntomas, es decir, como un epifenómeno de lo biológico. Lo anterior, presenta el riesgo de tomar a la enfermedad como una estructura fija que persiste transculturalmente, cuyo contenido es entendido en términos de elementos inarticulados, vacíos de significado, prescindibles en tanto que no explican o no pueden albergar la causa objetiva de la alteración del funcionamiento estructural. Tal práctica metodológica en su mayoría, culmina no sólo en la imposición de categorías sino en la deslegitimación de las expresiones lingüísticas y las formas que toma el padecer al interior de la región de estudio.

El uso de las categorías psiquiátricas como realidades más que como representaciones ha conducido al efecto definido por Kleinman como "falacia categorial" especialmente cuando estamos exportando nosologías y criterios diagnósticos a contextos culturales en donde éstos no adquieren coherencia ni sentido, o adoptan otras coherencias y otros sentidos (Martínez-Hernáez; 2006a: 2271).

De forma análoga a lo presentado previamente respecto al discurrir entre una concepción particularista y una universalista de la cultura, se puede distinguir en el campo de los trastornos mentales, el correr de perspectivas, entre la búsqueda y constatación de la invariabilidad de la enfermedad bajo la cual la particularidad cultural persiste al nivel de lo aparente. Como parte de ésta discusión en torno a la universalidad de la enfermedad mental, Uribe (2000) llama la atención respecto a la serie de estudios que la Organización Mundial de la Salud impulsó en la década de los años sesenta, a fin de mostrar la presencia de ciertos trastornos en todo el mundo, con lo cual quedarían afirmados como categorías universales y entidades independientes del sujeto que las vive. Entre estos trastornos son mencionados la 
Ensayo Especialización

Antropología de la Cultura

Natalia Bautista Aguilar esquizofrenia, la psicosis maníaco-depresiva, cuyo término se reformuló y actualmente es identificado en el Manual Diagnóstico y Estadístico de los Trastornos Mentales (DSM-IV) como trastorno afectivo bipolar, ciertos trastornos de ansiedad, trastornos orgánicos del cerebro y la depresión mayor. No obstante, se señala la enorme variación que ha mostrado la sintomatología, como en el caso de la esquizofrenia, dejando ver que hay algo más allá de la explicación neurofisiológica que interviene en ella. De acuerdo con Kleinman (1988) esto se debe a la existencia de "una ideología profesional tácita que exagera lo que es universal en la enfermedad mental, al punto de desechar lo que ella tiene de particular en términos culturales" (Kleinman 1988 cit. en Uribe, 2000: 348).

A lo largo de la historia del Manual Diagnóstico y Estadístico de los Trastornos Mentales (DSM) se muestra un cambio en la conceptualización de la salud mental y la enfermedad, dirigida a adherirse o ajustarse al canon de la biomedicina como una forma de dotar a la psiquiatría de una identidad médica. Sin embargo, la evolución de la clasificación misma ha mostrado ser variable en el tiempo, además de dependiente de cambios en las escuelas de pensamiento dominantes, donde cada edición va reflejando el espíritu de la época (DSM, 1952; DSM-II, 1968; DSM-III, 1980; DSM-III-R, 1987; DSM-IV, 1994; DSM-IV-TR, 2000 у DSM-5, 2013). Kawa y Giordano (2012) señalan cómo, trastornos que aparecían en la primera versión del Manual Diagnóstico publicada en 1952, los cuales eran tratados con enfoques psicoterapéuticos, para 1980, en la tercera versión, compartían la tendencia junto con demás trastornos a ser tratados con psicofármacos. Lo que a su vez impulsó el desarrollo de la industria farmacéutica focalizada a incidir en el aspecto biológico de la enfermedad. A este respecto, es importante llamar la atención sobre el aumento en el consumo de antidepresivos, en las sociedades industrializadas que Martínez-Hernáez (2006b) identificara como mercantilización de los estados de ánimo. Bajo la mirada de la industria farmacéutica quienes presentan trastornos mentales son vistos como consumidores, situación que merece un análisis profundo ya que si bien, ciertas condiciones psiquiátricas han mostrado la posibilidad de ser tratadas exitosamente 
Ensayo Especialización

Antropología de la Cultura

Natalia Bautista Aguilar

mediante fármacos, otras tantas no comparten el mismo resultado respecto a la recuperación.

A pesar de que la elaboración de la clasificación estandarizada de los trastornos mentales representó un avance en la investigación; ésta, ha conllevado también dificultades y abusos en la manera en que dichos trastornos han sido entendidos, diagnosticados y tratados. La discusión acerca de la aplicación universal de las categorías psiquiátricas, llevaron a que en 1991 se diera la revisión del Manual Diagnóstico y Estadístico vigente en ese momento (DSM-III-R), a fin de revisar el papel jugado por la cultura en los trastornos mentales. Dicho análisis culminó en la construcción de la categoría de los síndromes culturales o de filiación cultural (culture-bound syndromes) que fueron incluidos en la siguiente versión del Manual Diagnóstico (DSM-IV). Los síndromes culturales o de filiación cultural quedaron definidos de la siguiente forma:

\begin{abstract}
Patrones recurrentes de comportamiento aberrante y de experiencias problematizadoras con una expresión geográfica específica, que pueden estar no relacionados con las categorías diagnósticas del DSM-IV. Muchos de estos patrones son considerados localmente como "enfermedades", o por lo menos como aflicciones, y la mayoría reciben una denominación propia. No obstante que las presentaciones que conforman las principales categorías del DSM-IV aparecen en todo el mundo, los síntomas particulares, el curso de la enfermedad y la respuesta social son a menudo influenciados por factores culturales locales. En contraste, los síndromes culturales generalmente están limitados a sociedades o áreas culturales específicas, y representan categorías diagnósticas localizadas, "folk", que enmarcan significados coherentes para ciertos conjuntos de experiencia y observación repetitivos y generadores de dificultades (APA ${ }^{1}$, DSM-IV trad. Uribe, 2000: 349).
\end{abstract}

Detrás de tal definición, es posible identificar diferentes aspectos fundamentales, por un lado, algunos elementos de lo que en su momento constituyeran las propuestas de la escuela culturalista que veía a la cultura como una serie de comportamientos concretos de individuos que a su vez eran concebidos como propios de cada cultura, es decir, en los cuales recaía su particularidad, volviendo

\footnotetext{
${ }^{1}$ American Psychiatric Association (Asociación Americana de Psiquiatría)
} 
Ensayo Especialización

Antropología de la Cultura

Natalia Bautista Aguilar

correspondientes pluralidad cultural y pluralidad de personalidad, de estilos 0 tipos culturales. A pesar de la diversidad de trabajos y propuesta al interior de dicha escuela, la premisa reflejada en la anterior definición es aquella que otorga un cierto esencialismo a la cultura, que además es atribuida a un grupo cerrado y singular, es decir, propia de grupos específicos vistos como entidades separadas. No obstante, como pudo leerse en líneas anteriores, al interior del enfoque culturalista se dio la tendencia a superar tipologías fijas al concebir la posibilidad de diversidad al interior del propio grupo. Un enfoque que no apostaba por la estabilidad de las culturas sino que reconocía a los individuos como creadores, transmisores y transformadores de la misma, no como sujetos pasivos. De este modo, veía a la cultura en términos más dinámicos, no como algo 'dado' que el individuo recibe como una totalidad, de una vez y para siempre...y, de todas maneras, nunca puede adquirir toda la cultura de su grupo" (Cuche, 2002: 51).

En la definición de lo cultural en relación a tales síndromes, persiste la referencia a una cultura localizada, estática, que apunta al reforzamiento de la dicotomía urbano-rural que en muchos casos se proyecta en una escala más amplia de correspondencia a la oposición primer mundo/tercer mundo, "extremando así, las fronteras entre ambos entornos, "entre un supuesto "cosmopolitismo" frente a una supuesta "cultura", donde "la comunidad" se ha presentado como algo dado, como si fuera un sujeto individual" (Ortner, 1994 cit. en Camus, 2002:81). Por tanto, la inclusión de tales padecimientos (síndromes culturales), no subsana la marginación o secundarización de lo cultural en la enfermedad mental, por el contrario, fortalece su segregación a campos y sentidos particulares ya que, tales síndromes son definidos a partir de su presencia en contextos y poblaciones específicas, esto es, la incursión de lo cultural a partir de la oposición universal/particular, bajo la que son distinguidos aquellos trastornos con presencia transnacional, como la esquizofrenia o depresión y los síndromes culturales que se presentan en una particular cultura y que son considerados expresión de una condición específica de la persona que habita una zona o región particular. 
Ensayo Especialización

Antropología de la Cultura

Natalia Bautista Aguilar

Las críticas lanzadas desde el propio campo de la psiquiátrica reclaman que dicha especificidad cultural, los ha vuelto exóticos al señalarlos como una distintiva patológica de un "Otro" ajeno. Pareciera ignorarse que cada categoría diagnóstica requeriría de una consideración de los factores culturales que intervienen en la misma (Uribe, 2000). Es así que la principal discusión continúa recayendo sobre la configuración de un sistema clasificatorio de la enfermedad mental, tendiente a la estandarización de las culturas a costa de la cancelación de diferencias locales. De ahí que considero que en el campo de la investigación transcultural en salud mental, no debe seguirse trabajando sobre una visión esencialista de la cultura que reduzca la diferencia o intente pasarla por alto en nombre de una universalización de padecimientos o categorías.

Si bien, hay una serie de criterios médicos establecidos para la clasificación de los diversos trastornos mentales, lo cierto es que no hay una homogeneidad respecto a la configuración, interpretación y vivencia de éstos, a diferencia de otras enfermedades en las cuales puede encontrarse una regularidad en la expresión de síntomas. Mientras que para la medicina los padecimientos mentales se describen a través de diagnósticos clínicos, para las personas se encuentran interrelacionados a sus condiciones de vida que como tal integran la descripción, origen y posibilidad de recuperación de la misma. Por lo tanto, hablar de salud lleva implícita la referencia a aspectos no sólo biológicos sino a otras dimensiones del sujeto que aluden a una multiplicidad de factores como la interacción con el entorno y los postulados y condicionamientos socio-culturales al interior de los cuales los grupos sociales configuran la enfermedad y dotan de significado al proceso salud/enfermedad/atención, en su totalidad.

En el caso de México, ejemplos de lo anterior, pueden encontrarse en la literatura desde Aguirre Beltrán (1994) quien hizo mención del papel jugado por la angustia en el desarrollo de neurosis y psicosis en mestizos, indios y mulatos durante el periodo de colonización. La angustia para él, era una capacidad orgánica que participaba también del contexto social, a partir de desencadenarse debido a una 
Ensayo Especialización

Antropología de la Cultura

Natalia Bautista Aguilar

situación que el individuo experimenta como amenaza para su existencia y frente a la cual se visualiza impotente en su resolución. Es decir, el individuo pone en juego su referente cultural a través del cual ha aprendido a enfrentarse a diversas situaciones ante el contacto con otro grupo poseedor de otro sistema cultural. Sus observaciones en ese momento, lo llevaron a rastrear en el proceso de aculturación, causas del desencadenamiento de padecimientos mentales viendo la reacción ante la angustia como un instrumento proporcionado por la propia cultura, que llega a participar en el mantenimiento del orden social. Más recientemente, Ríos (2011) retrata la situación de algunos de los 140 trabajadores agrícolas mexicanos indocumentados que fueron deportados de Estados Unidos al presentar síntomas de alguna enfermedad mental en las décadas que van de 1920 a 1944. Lo que llevó a la construcción, por parte de psiquiatras mexicanos, de la categoría "psicosis del repatriado", que se caracterizaba por hablar incoherencias relacionadas a grandes negocios y riquezas en Estados Unidos, un aspecto físico descuidado, falta de apetito, apatía y desorientación en el espacio y tiempo; síntomas, que después de una buena dieta y descanso, desaparecían. Ello llevó a los médicos mexicanos a concluir que el síndrome no obedecía a factores bioquímicos sino a causas sociales (Ríos, 2011). Asimismo, otros trabajos, en este mismo esfuerzo por rescatar la dimensión sociocultural que participa en la configuración de la enfermedad mental, observan como entre los wixárika, la locura puede ser resultado de violar la norma social, "el costumbre" o bien, forma parte del rito de iniciación chamánica, a través del cual un Dios cuya materialización tiene lugar en algún ser de la naturaleza, pretende comunicar un don (Villaseñor y Aceves, 2003). O bien, entre tzotziles y tzeltales, se localizan referencias a comportamientos que implicaban alucinaciones, delirios y ataques, que se concluye responden a experiencias diversas vinculadas a las dimensiones de la vida social (Eroza y Álvarez, 2008).

Otros trabajos revisados por Duero y Shapoff (2009), muestran la influencia de factores sociales y culturales en el desarrollo y vivencia de enfermedad, algunos de éstos son: estudios longitudinales que sostienen que en Occidente el curso de 
Ensayo Especialización

Antropología de la Cultura

Natalia Bautista Aguilar

la enfermedad es menos favorable en comparación a otras sociedades (Alanen et al., 1986; Harding et al., 1987); así como, trabajos que señalan para el caso de familias mexico-americanas, que la identificación de la esquizofrenia como "nervios" lleva al uso de un lenguaje menos estigmatizante en torno al mismo, con lo que permitirían una mejor integración social de la persona enferma (Jekins s/f, citado en Kleinman, 1991). De igual manera, trabajos enfocados a trastornos como el de depresión mayor que postulan un riesgo menor de suicidio en los países del tercer mundo, en comparación con el riesgo en países desarrollados (Kleinman, 1991). Por otra parte, en un estudio realizado por Orquendo et al. (2000) con población caucásica, afroamericana y estadounidense de origen centroamericano, cubano y puertorriqueño residentes en Estados Unidos, se encontró que los hombres de origen puertorriqueño y las mujeres pertenecientes a los cinco distintos grupos de población, presentaban mayor tasa de depresión, sin embargo, correspondían a su vez, a los grupos que reflejaban la menor tasa de suicidio consumado. La razón de tal diferencia, se vinculó al nivel de integración familiar, expectativa del pronóstico y práctica religiosa como sistema de soporte social (Duero y Shapoff, 2009).

Todos éstos, pese a representar valiosos esfuerzos por realizar un análisis cultural de los trastornos mentales, no agotan el cuestionamiento sobre cómo son percibidos, experimentados y representados interculturalmente, el cual continúa siendo una interrogante de extendida validez, que se suma a la necesidad de trabajar en el reconocimiento de la agencia del sujeto, en su propio proceso de enfermedad, sobre todo en estudios en torno al suicidio. Además de ser hallazgos que no han sido estimados en su justo valor, dado que la práctica psiquiátrica continúa postulando una concepción esencialista y universalista de síntomas y enfermedades. En muchos otros casos, la propia investigación transcultural se basa en el supuesto de que "la biología determina el tipo de enfermedad y la cultura sólo configura "disfraces" al verdadero trastorno subyacente" con lo que "se expresa una tendencia a interpretar las distintas señales de malestar y sufrimiento como expresiones culturales diferentes de un mismo fenómeno" (Duero y Shapoff, 
Ensayo Especialización

Antropología de la Cultura

Natalia Bautista Aguilar

2009: 38). Cuando en realidad habría que reconocer que las categorías diagnósticas son formas de clasificar ciertos fenómenos a partir de criterios específicos que han sido convenidos por un grupo determinado de científicos que responden a intereses teórico-metodológicos particulares.

En términos de Leach (1981) podría decirse que tales categorías, organizan una serie de aspectos de la conducta y el pensamiento en conjuntos estructurados dentro de los cuales, cada uno de los comportamientos descritos, toman el lugar de signos y síntomas cuyo valor es adquirido en relación con los otros. "Los signos no se presentan aislados; un signo es siempre miembro de un conjunto de signos contrastados que funcionan dentro de un contexto específico; un signo sólo transmite información cuando se combina con otros signos y símbolos del mismo contexto" (Leach, 1981: 19). Por tanto, los criterios señalados en el DSM, no tienen sentido por sí solos, son signos que entran en combinación o relación con los demás al interior de marcos de significados y contextos específicos.

Así, otro de los problemas que se desprende de la pretensión de universalidad de la ontología psiquiátrica es el concerniente a la medición, traducción y comparación de experiencias y sistemas de representación de la realidad de las diferentes culturas. Dicho problema se presenta en su forma más clara en el ámbito de las investigaciones que abordan la presencia de trastornos mentales en diversas sociedades a partir de la aplicación de instrumentos de medición estandarizados. Esta metodología en el caso de países como México, representa un desafío debido a la diferencia de idioma, ya que en su gran mayoría dichos instrumentos reconocidos como válidos en el ámbito académico, han sido construidos en sociedades de habla inglesa, de ahí que la traducción y adaptación denominada transcultural se haya constituido como el primer paso de toda investigación que pretenda trabajar de ese modo. La búsqueda de equivalencia implica la traducción no sólo de palabras sino de ideas que resulten familiares a los nuevos lectores que no pertenecen a la cultura o contexto en que originalmente fue escrito el texto en cuestión (Burke, 2010). No obstante, no existe 
Ensayo Especialización

Antropología de la Cultura

Natalia Bautista Aguilar

un consenso acerca de la forma ideal para realizar la traducción y ni siquiera en algunos casos hay un consenso respecto a lo que eso significa. Culminando en investigaciones que tan sólo se circunscriben a traducir de forma literal los instrumentos a utilizar sin ocuparse de la adaptación profunda al contexto social y cultural en que pretende aplicarse. Cabe tener presente que en el caso de trastornos mentales no sólo se trata de una traducción literal de terminología sino de experiencias y emociones constituidas en síntomas que en principio pasan por una descontextualización para su recontextualización en la nueva población. Se trata de dimensiones de la cultura que no se pueden aprehender mediante la aplicación de instrumentos o mediciones cuantitativas y que en cuestión de salud mental resultan de vital importancia debido a que forman parte significativa del padecimiento. Dimensiones que se encuentran en el relato del propio paciente constituyendo narrativas que cuando no encuentran sustento físico-orgánico sólo dependen de la interpretación médica. Como Burke (2010) señala, la equivalencia de términos no da cuenta de toda la densidad de aquello que se busca traducir, además de que perdura la interrogante respecto a los elementos y sentidos que no pueden traducirse, y que terminan por constituir pérdidas culturales. De este modo, tanto la traducción como la interpretación, se constituyen como procesos de negociación de significados (Burke; 2010) al que la noción de poder no es ajena.

En el intento por responder a cuestionamientos sobre quién usa el lenguaje y en qué circunstancias, Wolf (2003) ubicaba la apertura al estudio del papel contextual del poder en el uso del lenguaje, que a su vez, guarda estrecha vinculación con la propuesta de Bourdieu (s.f. cit. en Wolf, 2003) de pensar la comunicación operando dentro de mercados lingüísticos en los cuales no todos los participantes ejercen el mismo control sobre el proceso, sino de acuerdo a su posición social. Para Bourdieu el lenguaje es un instrumento de poder y la competencia el derecho a hablar, al lenguaje legítimo y autorizado; el poder de imponer una recepción. El acto de habla en sí carecería de poder, a menos que quien lo realice esté autorizado institucionalmente, es decir, sea realizado por alguien reconocido culturalmente para ello (Bourdieu s.f. cit. en Wolf, 2003). Al interior del campo de la 
Ensayo Especialización

Antropología de la Cultura

Natalia Bautista Aguilar

salud mental y la salud en general, dicha autoridad o legitimidad se coloca del lado del profesional de la salud, al que se reconoce como especialista, en contraposición a la población general, incluidos los propios pacientes, a quienes se califica de ignorantes o poseedores de saberes equivocados que hay que erradicar en la mayoría de ocasiones. El discurso biomédico se ha constituido como el saber legítimo de la enfermedad, sin embargo, no debe olvidarse, que la psiquiatría es un conjunto de saberes y prácticas, entre otros, que dan significado a la misma, mediante el uso de categorías que son dependientes de un particular contexto histórico-cultural (Martínez-Hernáez; 2009). Asimismo, en la práctica concreta como parte de la investigación transcultural, en la traducción de instrumentos la noción de poder puede constatarse, no sólo en la hegemonía ya reflejada en el origen e idioma del instrumento, sino como subyacente al papel del traductor que es quien decide el manejo de los matices culturales que dará a su traducción, así como, al papel del investigador como quien define finalmente, el proceso al que será sometido el instrumento (sólo traducción de idioma o panel de expertos, retrotraducción, etc.) y las sugerencias o modificaciones que se integrarán.

Sobre el rol del médico, puede igualmente observarse como en el DSM-IV, a la par, que se busca establecer categorías objetivas y universales, se reconoce como fundamental el juicio de este, durante la evaluación clínica, en tanto que se constituye como el responsable de discriminar la presencia o no de un trastorno. "Las categorías y criterios diagnósticos sirven como guías que han de usarse partiendo del juicio clínico y que deben ser utilizados por personas con experiencia clínica" (APA, 1995 cit. en Duero y Shapoff, 2009: 32). De ese rol de experto, en quien recae la asignación de diagnósticos y tratamientos, también se desprende la inevitable consideración del sistema cultural del que proviene el mismo y que puede influir en la valoración de la persona y la identificación del diagnóstico, en la relación médico/paciente, así como en el tratamiento. Estudios comparativos señalan el diagnóstico variable que asignan los psiquiatras a personas con diferentes rasgos étnicos, por ejemplo, en ocasiones tienden a diagnosticar a 
Ensayo Especialización

Antropología de la Cultura

Natalia Bautista Aguilar

personas pertenecientes a minorías étnicas (hispanos, afroamericanos, etc.) con trastornos más graves o su comportamiento se califica como conducta delictiva en comparación con personas blancas (Duero y Shapoff, 2009). Representaciones socioculturales, conforman en muchas ocasiones, representaciones técnicas ya sea en forma total $o$ en una cierta medida, pero pueden estar presentes (Menéndez, s/f).

Ese enorme peso al juicio del psiquiatra ha dado por resultado que los actuales esfuerzos se dirijan a buscar lo que se ha denominado "competencia cultural" tanto en la fase de formación profesional del psiquiatra como a lo largo del proceso de atención. Ya no solamente como una estrategia para potencializar el alcance de la clasificación diagnóstica; sino como una forma de enfrentar también los nuevos retos en la atención que representan los constantes flujos de migración y que provocan una fuerte presencia de población inmigrante que llega a tener posibilidad de hacer uso de los servicios de salud. La competencia cultural se postula como un entrenamiento al que sería sometido el personal de salud, a fin de conocer los valores, creencias y prácticas de los diversos grupos étnicos a los que pudieran llegar a pertenecer las personas que los consulten y proporcionar servicios culturalmente adecuados (Betancourt, 2002; Bhui et al. 2007). Sin embargo, como puede observarse, en el enfoque de la competencia cultural continúa existiendo una visión de cultura reducida a un conjunto de atributos o características, nuevamente esencialista y estática, que es sinónimo de pertenencia étnica y en muchos casos, fundamentada únicamente en la diferencia lingüística, sin prestar atención en la forma en que la cultura interviene en la configuración de la persona y su percepción de enfermedad, su vivencia y tratamiento. Además de que reduce lo cultural a entrenamiento y una especie de habilidad que recaería en el médico quien finalmente, lo emplearía como una estrategia para la interacción dirigida a la inserción del paciente a la lógica biomédica.

Unas pocas horas de educación 'antropológica' no van a modificar los saberes médicos cuya formación se caracteriza cada vez más por ser 
Ensayo Especialización

Antropología de la Cultura

Natalia Bautista Aguilar

fuertemente biomédica (.....) Hay toda una serie de características de las orientaciones biomédicas que obedecen a condiciones institucionales y profesionales que no sólo se pueden modificar con mayor información/educación (Menéndez, 2006: 59).

Autores como Kleinman (1987) sostienen que la atención clínica debería proporcionar idealmente un tipo de mini-etnografía, culturalmente sensible, de los pacientes de forma que abarque las metáforas culturales y personales. Lo anterior, encuentra eco y trascendencia en la idea de interculturalidad en los términos que Mato (2009) establece, esto es, como una herramienta para el análisis de las relaciones de colaboración, confrontación o conflicto, entre agentes sociales que se perciben culturalmente diversos respecto de cualquier referente, y no sólo el étnico, que resulte significativo; que a su vez incluya valores de reconocimiento y respeto mutuo. En México, la aplicación del concepto de interculturalidad en el campo de la salud en ocasiones ha caído en lo que Mato (2009) denomina interculturalismo, es decir, con una orientación de pensamiento y acción que alude a programas o políticas públicas en torno a la salud que continúan enfocadas a la modificación y desplazamiento de las creencias y prácticas opuestas al modelo biomédico. Definir la diferencia sólo por el aspecto étnico, termina por cargar de un sentido segregacionista a la aplicación del término. A este respecto, mantiene vigencia el desafío de reflexionar y construir estrategias que permitan abordar el estudio de la diferencia y desigualdad, no sólo cultural sino social, económica y política que impacta la configuración de los padecimientos.

A lo largo de este trabajo, la intención ha sido mostrar la dificultad de hablar de la cultura en los términos que la biomedicina lo hace, aplicándola al establecimiento de categorías fijas ante formas distintas de concebir, experimentar y nombrar objetos, situaciones, o en este caso, malestares y padecimientos. Así como, lo inadecuado de establecer una noción homogénea y esencialista de la cultura, al interior del proceso de salud/enfermedad/atención, debido a que proceder de ese modo, anula la posibilidad de revertir la asimetría entre saberes, y promueve la incomunicabilidad entre sistemas de pensamiento, al sostener como único y válido a uno en particular. Una característica reconocida al relativismo cultural, es aquella 
Ensayo Especialización

Antropología de la Cultura

Natalia Bautista Aguilar

de resaltar la importancia de erradicar todo etnocentrismo que lleve a la imposición de paradigmas de alcance universal con carácter de "superiores" (García Canclini, 1982). Premisa, que en el campo de la salud/enfermedad mental/atención, sigue siendo necesario atender a fin de superar imposiciones y referentes de superioridad, que han caído en lo que Cuche (2002) señala como conversión del derecho a la diferencia en asignación de la misma. Habría pues, que entender a las clasificaciones actuales de los trastornos mentales, sostenidas por el modelo biomédico, como constructos y no como hechos indiscutibles.

El análisis cultural conserva actualmente toda su pertinencia y sigue siendo apto para dar cuenta de las lógicas simbólicas instauradas en el mundo contemporáneo, siempre que no se dejen de lado las enseñanzas de las ciencias sociales. No basta con tomar de ellas la palabra "cultura" para imponer una lectura de la realidad que, a menudo, oculta un intento de imposición simbólica (Cuche; 2002:8).

Lo presentado al inicio de este ensayo en torno al uso y construcción de la noción de cultura, mantiene correspondencia con lo descrito por García Canclini (2004) como las principales narrativas que persisten cuando se habla de cultura, por un lado, la persistencia de una concepción de aplicación cotidiana del término como referente de educación, refinamiento, cúmulo de conocimientos, que al día de hoy persiste en el imaginario de amplios sectores de la población y que guarda un efecto importante en la interacción social. Por el otro, en oposición a ese uso cotidiano, un uso configurado desde el ámbito científico que buscó integrar tantas dimensiones de la vida social como intereses teórico-metodológicos disciplinarios. En relación con ese uso científico, si bien, desde la antropología simbólica con Geertz (1973) se había señalado ya la importancia de los símbolos como elementos de definición de la cultura; Camus (2002) precisa que tal matriz de significados que sería la cultura, resulta más fácil de ser aprehendida y hacerla visible en tanto que se aterriza en un entramado social. De esta forma, el alcance de una posible definición operativa de la cultura, que a su vez llegara a ser compartida por autores y disciplinas, quedaría situada al afirmarla como el "conjunto de los procesos sociales de significación", esto es, "procesos sociales de producción, circulación y consumo de la significación en la vida social" (García 
Ensayo Especialización

Antropología de la Cultura

Natalia Bautista Aguilar

Canclini, 2004: 34). Esta perspectiva representa el paso de una visión estática de elementos a una de proceso, en el que tales elementos se conciben creados y recreados continuamente al interior de la historia social, colocando el énfasis en el análisis intercultural de los significados que son recibidos, reprocesados 0 recodificados, sin perder de vista las relaciones de poder que los cruzan; una "perspectiva procesual que considera a la vez lo sociomaterial y lo significante" (García Canclini; 2004: 35) de la misma.

El pensar la cultura en estos términos, en el caso de los trastornos mentales, lleva a dar cuenta tanto de las condiciones histórico-sociales que sirven de contexto, como de las fuerzas y relaciones de poder que fundamentan la emergencia de los discursos sobre la enfermedad mental. Así también, posibilita profundizar en la dimensión cultural de toda práctica social, tanto de quien presenta un trastorno, como de la propia práctica médico-psiquiátrica. Como se mencionó en el cuerpo del texto, es importante prestar atención a la posición que ocupa la industria farmacéutica en la configuración de las clasificaciones diagnósticas y asignación de tratamiento médico, a través de su participación mediante el financiamiento de investigaciones, de revistas, congresos y reuniones científicas, de la difusión de información, entre otras. Una industria que si bien, ha impulsado el desarrollo de la medicina con beneficios para la población, alberga intereses respecto al consumo y se ubica entre las principales industrias a nivel mundial en términos de concentración de capital (Martínez-Hernáez, 2006b).

Asimismo, tal sentido de lo cultural constituye una vía de acercamiento al reconocimiento de la perspectiva del sujeto, en este caso la persona que tiene el trastorno, considerando a su vez, la capacidad de agencia de los diferentes actores involucrados en el proceso salud/enfermedad/atención. De esta forma, considero constituye una herramienta teórico-metodológica pertinente para el análisis del proceso aquí señalado, como aquella que rescata la dimensión contextual, heurística y comparativa de la noción de cultura, señalada por Appadurai (2001), bajo la cual, hablar de lo cultural quedaría constituido como la 
Ensayo Especialización

Antropología de la Cultura

Natalia Bautista Aguilar vía para la trascendencia de la sustantivación de la misma en el terreno de los trastornos mentales. Una perspectiva desde la cual el análisis cultural de los mismos, tendría que atender a los procesos de interacción, confrontación y negociación que tienen lugar entre grupos y sistemas culturales diversos (García Canclini; 2004).

La evidencia de la articulación entre factores sociales, culturales y biológicos, no es escasa y responde a una larga trayectoria de estudio y discusión, no obstante, atendiendo a lo señalado por Menéndez (s/f), respecto a que una de las cuestiones básicas, no es tanto el seguir simplemente evidenciando el papel de lo cultural en la configuración de los trastornos, sino en profundizar el análisis en torno al por qué la biomedicina, y en particular la psiquiatría, continúan prestando escasa atención a tales hallazgos, optando por excluirlos o medicalizarlos. El análisis de estos procesos (interacción, confrontación y negociación), cobran importancia en tanto contribuyen a la identificación de los posibles elementos o dinámicas que permitirían sentar las bases de posibles respuestas a tal cuestionamiento.

Finalmente, tener presente la idea de interculturalidad como un objeto de estudio revelador y cuestionador (García Canclini, 2004) no sólo lleva al antropólogo a convertirse en especialista de las intersecciones, sino, que al constituirse como una forma de pensar la diversidad en los espacios de frontera entre sociedades distintas o bien, entre grupos o sujetos culturalmente diversos al interior de una misma sociedad. Permitiría dar paso a la configuración de un campo de estudio del fenómeno de la enfermedad mental orientado, más que por una perspectiva transcultural que apunte a la homogeneización de experiencias, por un enfoque dirigido al estudio de la experiencia de enfermedad mental en la interculturalidad que brinde luz a la comprensión de elementos que han sido ignorados o insuficientemente profundizados. 


\section{Ensayo Especialización \\ Antropología de la Cultura \\ Natalia Bautista Aguilar}

\section{Referencias}

Aguirre Beltrán, Gonzalo (1994). Antropología Médica. Sus desarrollos teóricos en México. Fondo de Cultura Económica. México.

Appadurai, Arjun (2001). La modernidad desbordada. Dimensiones culturales de la globalización, Argentina, Ediciones Trilce, Fondo de Cultura Económica.

Baldwin, John R., Faulkner, Sandra L. y Jecht, Michael L. (2006). "A moving Target: The Illusive Definition of Culture", en John R. Baldwin, Sandra L. Faulkner, Michael L. Hecht y Sheryl L. Lindsley (eds.), Redefining Culture. Perspectives Across the Disciplines, Lawrence Erlbaum Associates, New Jersey.

Betancourt, Joseph R., Alexander R. Green y J. Emilio Carrillo (2002). Cultural Competence in health care: emerging frameworks and practical approaches. Field Report, The Commonwealth Fund.

Bhui, Kamaldeep, Nasir Warfa, Patricia Edonya, Kwame McKenzie y Dinesh Bhugra (2007). Cultural competence in mental health care: a review of model evaluations, BMC Health Services Research, vol. 7, núm. 15. Disponible en http://www.biomedcentral.com/1472-6963/7/15. [Consultado el 20 noviembre de 2012].

Burke, Peter (2010). Hibridismo cultural. España, Akal.

Camus, Manuela (2002). Ser indígena en la ciudad de Guatemala. Guatemala. FLACSO.

Cuche, Denys. La noción de cultura en las ciencias sociales, Buenos Aires, Nueva Visión, Colección Claves, 2002

Duero, Dante G y Virginia Shapoff (2009). "El conflicto nosológico en psicopatología: notas críticas sobre el diagnóstico psiquiátrico", en Revista CES Psicología, vol. 2, núm. 2, pp. 20.48.

Eroza, E. y Álvarez G., G. C. (2008). "Narrativas del padecimiento "mental": arenas de debate en torno a prácticas sociales y a los valores que las sustentas entre los mayas tzotziles y tzeltales del sur de México", en Revista de Antropología Social, núm. 17, pp.377-410.

García Canclini, Néstor (1982). Las culturas populares en el capitalismo. Editorial Nueva Imagen. México.

García Canclini, Néstor (2004). Diferentes, desiguales y desconectados. México, Gedisa.

Geertz, Clifford (1973). La interpretación de las culturas. Trad. Alberto L. Bixio. España, Gedisa.

Kawa, Shadia; Giordano, James (2012). "A brief historicity of the Diagnostic and Statistical Manual of Mental Disorders: Issues and implications for the future of psychiatric canon and practice", en Philosophy, Ethics, and Humanities in Medicine, vol. 7, no 2. Disponible en http://www.pehmed.com/content/7/1/2 [Consultado el 15 de enero de 2013]

Kleinman, Arthur (1987). "Anthropology and psychiatry. The role of culture in a cross-cultural research on illness", en The British Journal of Psychiatry, núm. 151, pp. 447-454.

Leach, Edmund (1981). Cultura y comunicación. La lógica de la conexión de los símbolos. Editorial Siglo XXI de España. 


\section{Ensayo Especialización \\ Antropología de la Cultura \\ Natalia Bautista Aguilar}

Lévi-Strauss, Claude (1983). Las estructuras elementales del parentesco. Trad. Marie Therése Cevasco. México, Editorial Paidós.

Martínez-Hernáez, Ángel (2006a). "Cuando las hormigas corretean por el cerebro: retos y realidades de la psiquiatría cultural”, en Cadernos de Saúde Pública, vol. 22, núm. 11, pp. 22692280.

Martínez-Hernáez, Ángel (2006b). "La mercantilización de los estados de ánimo. El consumo de antidepresivos y las nuevas biopolíticas de las aflicciones", en Política y Sociedad, vol. 43, núm. 3, pp. 43-56.

Martínez-Hernáez, Ángel (2009). "Psiquiatría cultural. Teorías, comparaciones y políticas en salud mental”, en A. Martorell, J.M. Comelles y M. Bernal (eds.) Antropología y enfermería. Campos de encuentro. Un homenaje a Diana Garcés, II. Terragona, España, pp.143-165.

Mato, Daniel (2009). "Contextos, conceptualizaciones y usos de la idea de interculturalidad", en Miguel Ángel Aguilar, Eduardo Nivón, María Ana Portal, Rosalía Winocut (coords.) Pensar lo contemporáneo: de la cultura situada a la convergencia tecnológica. México, Anthropos, Universidad Autónoma Metropolitana (Pensamiento Crítico/Pensamiento Utópico 184).

Menéndez, Eduardo L. (2006). "Interculturalidad, 'diferencias' y Antropología "at home”. Algunas cuestiones metodológicas", en Gerardo Fernández Juárez (coord.) Salud e interculturalidad en América Latina. Antropología de la Salud y Crítica Intercultural, Quito, Ecuador, Ediciones AbyaYala.

Menéndez, E. L. (s/f). Los otros y nosotros: La parte negada de la cultura. Disponible en: http://documentacion.aen.es/pdf/libros-aen/coleccion-estudios/salud-mental-diversidad-ycultura/parte1-salud-mental-globalizacion-y-exclusion/029-los-otros-y-nosotros-la-parte.pdf [Consultado el 20 noviembre de 2012].

Ortner, S. B. (1993). "La teoría antropológica desde los años sesenta". Guadalajara: Universidad de Guadalajara (Cuadernos de antropología).

Ríos Molina, Andrés (2011). "La psicosis del repatriado. De los campos agrícolas en Estados Unidos al Manicomio La Castañeda en la ciudad de México, 1920-1944”, en Mexican Studies, vol. 27, núm. 2, pp. 361-384.

Uribe, Carlos Alberto (2000). "La controversia por la cultura en el DSM-IV", en Revista Colombiana de Psiquiatría, vol. 29, núm. 4, pp. 345-356.

Villaseñor B., Sergio J. y Aceves P., Martha P. (2003). "Maxayiákame. El concepto de 'enfermedad mental' entre los huicholes de Tuxpan de Bolaños, Jalisco México, en Revista Universidad de Guadalajara, núm. $30 . \quad$ Disponible en http://www.cge.udg.mx/revistaudg/rug30/babel30maxayiakame.html [Consultado el 10 junio de 2010].

Wolf, Eric (2003). Figurar el poder. México, Centro de Investigaciones y Estudios Superiores en Antropología Social. 